\title{
S-R separation with monkeys ${ }^{1}$
}

\author{
DOUGLAS L. MEDIN ${ }^{2}$ \\ UNIVERSITY OF SOUTH DAKOTA
}

Four monkeys were tested on successive problems involving cue-response separations. The usual $A A$ go left/BB go right paradigm was altered by introducing neutral $(N)$ stimuli in place of one of the As or $B s$ to create $N A, A N, B N$, and $N B$ configurations. The neutral stimulus created cue-response separations equal to the foodwell separation in two of the configurations ( $N A$, and $B N$, where $A$ signals go left and $B$ signals go right). Three of the four monkeys were able to solve these cue-response separation problems. Performance was discussed in terms of observing response theory and with respect to differences between the simultaneous and successive problem paradigms.

There is a considerable body of literature which indicates that primate discrimination performance varies inversely with spatial separation between cue and response (Meyer et al, 1965). Stollnitz (1965) suggested that these data could be accounted for by observing response theory. An observing response is any response that results in exposure to a discriminative stimulus (Wyckoff, 1952). Stollnitz assumed that the probability of an observing response varied inversely with cueresponse separations and poor performance on problems with cue-response separation was attributed to the lack of Ss making appropriate observing responses.

Performance deficits may also be reflected in the fact that, with an opportunity to make an observing response, $\mathrm{S}$ must also be able to respond appropriately on the basis of it. Davis et al (1964) tested monkeys in a situation where a pointer indicated the correct response. Monkeys were required to respond to the foodwell closest to the pointer which itself varied in position from trial to trial. Although performance was above chance level for all pointer positions (except where the pointer bisected the distance between the foodwells), monkeys made increasingly more errors with increasing cue-response separations. This was true even after 1300 trials which should have been long enough to allow the development of appropriate observing responses.

The two present experiments were designed to examine the effects of cue-response separation in the successive or sign-differentiated position problem (AA go left/BB go right) paradigm. In the successive paradigm there should be less response ambiguity than in the simultaneous discrimination paradigm given that an observing response has been made.

\section{Experiment 1}

Method. The Ss were four young but relatively sophisticated laboratory reared rhesus monkeys (Macaca mulatta). All had prior experience with object quality discriminations, but none had received experience with successive problems.
Testing was conducted in a WGTA on a formboard with foodwells 12 in. apart center-to-center. Stimuli were 2 in. diameter discs constructed of $1 / 2$ in. plywood.

Procedure. The monkeys were given the successive problem in the following fashion. For two Ss a red disc covering each foodwell (RR) indicated that a response to the righthand foodwell was correct, and two yellow discs (YY) indicated that a response to the lefthand foodwell was correct. For the other two Ss reward conditions were reversed. The white discs were considered neutral and were used to create the separation conditions. Thus a red and a white disc paired (RW or WR) indicated a response to the right as correct for two Ss, and a yellow and a white disc paired ( $Y W$ or WY) indicated a response to the left as correct. The RW (red disc over left foodwell, white disc over right foodwell) or the WY condition represents cue-response separations of approximately $10 \mathrm{in}$. and requires Ss to utilize information from the foodwell opposite the response site to achieve proficient performance. The six possible stimulus conditions were grouped into three types: double cue (YY, RR), single cue without cue-response separation (WR and YW if red signals go right and yellow signals go left), and single cue with cue-response separation ( $R W$ and WY if the reward is in the right and lefthand foodwells, respectively). Each condition occurred in a random order eight times each day for 15 days. Analysis of variance was performed (for both experiments), and where effects were significant $(p<.05$ or better), multiple comparisons were made.

Results. Performance improved significantly with practice (five day periods) for all three types of presentation $(p<.05)$. Examination of individual performances showed that three of the four monkeys were able to utilize information above the foodwell opposite the response site within 15 days of practice. Stimulus presentation conditions were not significantly different. One problem with the design of Experiment 1 is that the Ss might have responded to single cue conditions (RW, WR, YW, WY) as if they were simultaneous discriminations. Indeed for the first five day period, three of the four Ss made significantly below chance errors on the single cue/no separation condition and, correspondingly, significantly above chance errors on the single cue/separation condition; i.e., they treated the single relevant cue condition as a simultaneous discrimination. Experiment 2 was an attempt to minimize this interference effect.

Experiment 2

Method. The same Ss and apparatus were used in 
the second experiment. Green, blue, and white discs 2 in. in diameter and constructed of $1 / 2 \mathrm{in}$. plywood were employed.

Procedure. The Ss were initially trained on a simple GG go right (or left) $\mathrm{BB}$ go left (or right) successive problem. The monkeys were tested until they reached the criterion of $90 \%$ or better correct. For critical testing the single cue conditions (BW, WB, GW, WG) were introduced for 16 of the 40 daily trials, and 15 days of testing were given. Again the solution was green go right, blue go left for half the Ss and green go left, blue go right for the other half.

Results. The stimulus conditions were grouped into double cue, single cue without separation, and single cue with separation as in Experiment 1. The double cue condition resulted in the best performance, the single cue without separation condition resulted in intermediate performance levels, and the single cue with separation condition resulted in the worst performance. However, the performance of three of the four monkeys on the single cue with separation condition was significantly above chance $(80 \%)$ after the second five day block and reached $90 \%$ by the end of the experiment. Performance improved with practice periods only for the single cue with separation condition. One monkey made significantly $(\mathrm{p}<.05)$ more errors than chance on the single cue with separation condition for each practice period, but this was not the same $S$ that had failed on the Experiment 1 separation condition. The fact that this same $S$ also made fewer than chance errors on the single cue without separation condition for each practice period indicated that it had treated the problem as a simultaneous color discrimination (as has already been discussed in Experiment 1). This was true in spite of the fact that this strategy results in 50\% errors.

Discussion

Three of four monkeys in both experiments provided clear evidence of being able to utilize information remote from the response site in solving successive discrimination problems. Two factors may be suggested as responsible for this performance. First, the probability of an observing response may be high at foodwell sites even if the foodwell is not the appropriate response site (as in the separation condition). Given this high initial probability of an observing response, one might expect the monkeys to be able to solve this 10 in. (edge to edge) cue-response separation. French et al (1965) reported findings which indirectly support this idea.

A second explanation for the relatively proficient performance may lie in the successive paradigm itself. In the successive paradigm a cue signals an appropriate positional response, while in a simultaneous discrimination the appropriate response is to a particular stimulus rather than to a position. Given high probabilities of an observing response, one would expect performance on a successive problem to be relatively insensitive to cue-response separations. With cue-response separations in a simultaneous discrimination, the $S$ may attend to the cue but be unable to judge the appropriate response site. Therefore one would expect simultaneous discrimination problems to be more sensitive to cue-response separations than successive problems. This idea is supported by work with rats (Weise \& Bitterman, 1951; Bitterman \& Wodinsky, 1953) and with children (Lipsitt, 1961), where performance was found to be better under simultaneous than successive conditions with no cue-response separation and better under successive than simultaneous conditions when cue and response were separated.

References

BITTERMAN, M. E., \& WODINSKY, J. Simultaneous and successive discrimination. Psychol. Rev., 1953, 60, 371-376.

DAVIS, R. T., LAMPERT, A., \& RUMELHART, D. E. Perception by monkeys. II. Use of cues at a distance by young and old monkeys. Psychon. Sci., 1964, 1, 107-108.

FRENCH, G. M., BIRNBAUM, I., LEVINE, R., \& PINSKER, H. Discriminative choice in normal and prefrontal rhesus monkeys. J. comp. physiol. Psychol., 1965, 59, 225-230.

LIPSITT, L. P. Simultaneous and successive discrimination learning in children. Child Develpm., 1961, 32, 337-347.

MEYER, D. R., TREICHLER, R. R., \& MEYER, P. M. Discrete training tecliniques and stimulus variables. In A. M. Schrier, H. F. Harlow and F. Stollnitz (Eds.), Behavior of nonhuman primates; modern research trends. New York: Academic Press, 1965.

STOLLNITZ, F. Spatial variables, observing responses and discrimination learning sets. Psychol. Rev., 1965, 72, 247-261.

WEISE, P., \& BITTERMAN, M. E. Response selection in discrimination learning. Psychol. Rev., 1951, 58, 185-195.

WYCKOFF, L. B., JR. The role of observing response in discrimination learning: Part I. Psychol. Rev., 1952, 59, 431-442.

Notes

1. This investigation was supported by USPHS Research Grant MH$07147-04$, Institute of Mental Health, to Dr. Roger T. Davis who supervised this project.

2. The author was an NASA predoctoral research fellow. 